\title{
A New Method for converting Slant lonospheric Delays to Vertical for SBAS lonospheric corrections
}

\author{
Bongkwan $\mathrm{Choi}^{1}$, Deokhwa $\mathrm{Han}^{1}$, Hwigyeom $\mathrm{Kim}^{2}$, and Changdon Kee ${ }^{1, *}$ \\ ${ }^{1}$ School of Mechanical and Aerospace Engineering and the SNU-IAMD, Seoul National University, Seoul, South Korea \\ ${ }^{2}$ School of Computing, KAIST, Daejeon, South Korea
}

\begin{abstract}
SBAS gives ionospheric correction data to GNSS users for more accurate positioning. SBAS uses obliquity factor of thin shell model to convert slant ionospheric delays to vertical delays which is not precise especially at lower elevation angle. This paper suggests a new method for estimating the vertical delays which are needed for generating the ionospheric corrections. The new method uses Chapman profile assumption by which the vertical electron density distribution can be modeled. It is also assumed that Chapman profile's parameters are given and the vertical ionospheric delay can be linearly modeled. We divided ionosphere into multi-layer and mapped ionospheric vertical delays with a linear function. Converting vertical delays of each layer to slant delays, we can set a linear equation for the vertical ionospheric delay at IPP. We used IRI model to get information about ionosphere for simulation to verify our new method. Estimation error of vertical ionospheric delay at IPP was decreased about $40 \%$ in average when using our new method.
\end{abstract}

\section{INTRODUCTION}

Global Navigation Satellite System (GNSS) is a system by which a user can point the user's location. The user uses receiver to receive signals from GNSS satellite and can calculate the position using data of signals. However there are many errors in space, such as ionospheric error, tropospheric error, satellite clock/orbit error, which make inaccuracy of estimation of the user's location. A major error among them is the ionospheric error. To mitigate ionospheric errors in GNSS signal, the user can use ionospheric models such as Klobuchar or get information about ionospheric delay from a reference station. Satellite Based Augmentation System (SBAS) is also the way to reduce the error of ionosphere. SBAS generates correction data which helps user to locate user's position more precisely. One of the correction data is the ionospheric correction data. SBAS gives ionospheric correction data of Ionospheric Grid Point (IGP) and the user uses the information of IGP to calculate user's ionospheric delay. The correction data of ionospheric delay at IGP is generated with the slant ionospheric delays in signal between satellites and reference stations. Master stations of SBAS need vertical ionospheric delays at Ionospheric Pierce Point (IPP), instead of slant delays, to calculate ionospheric correction data at IGP. Each reference station can measure the slant ionospheric delays with dual frequency, but cannot get the vertical ionospheric delays directly. As a result, reference stations have to estimate the vertical ionospheric delay and SBAS uses obliquity factor of thin shell model to get vertical ionospheric delays. However, thin shell model has some limitations.
First, thin shell model assumes that peak electron density height is constant. However the peak height is high at daytime and low at night time [1]. Furthermore thin shell model does not consider spatial characteristics of ionosphere. These factors occur errors in estimation of vertical ionospheric delay from slant ionospheric delay. In this paper, we suggested a new method for converting slant ionospheric delays to vertical ionospheric delay to mitigate the error of thin shell model.

\section{Algorithm of the new method}

In the new method we made several assumptions. First, we assumed that the variation of ionosphere is linear. We also assumed that the vertical electron density distribution follows Chapman profile [2]. Chapman profile is a model for electron density distribution of ionosphere. The function of Chapman profile is below.

$$
\begin{aligned}
& n_{e}(\lambda, \phi, h)=\frac{N_{0}(\lambda, \phi)}{\sqrt{2 \pi e} H} \exp \left(\frac{1}{2}(1-z-\exp (-z))\right)=N_{0}(\lambda, \phi) \operatorname{Chap}(h) \\
& \mathrm{Z}=\frac{h-h_{0}}{H} \\
& \int \operatorname{Chap}(h)=1
\end{aligned}
$$

$n_{e}$ is electron density at height $\mathrm{h}, N_{0}$ is the peak electron density, $h_{0}$ is the peak electron density altitude and $\mathrm{H}$ is the atmospheric scale height. The function of Chapman profile can be determined with two parameters $h_{0}, \mathrm{H}$ and we assumed that parameters of Chapman profile can be known at every point as a priory information.

Corresponding author: kee@snu.ac.kr 
Hoque suggested a method for estimating slant ionospheric delay in signal between a user and satellites with vertical ionospheric delay [3]. Fundamentally, the new method which is suggested in this paper started from the method in the paper of Hoque. The most different thing of the method of this paper from the Hoque's is that the process of the algorithm is inversed.

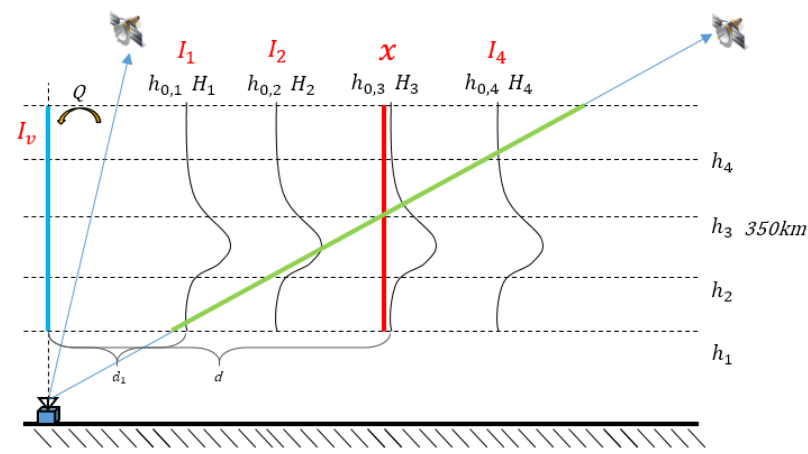

Figure 1. Conceptual figure of the new method

$$
\begin{aligned}
& I_{i}=\left(x-I_{v}\right) \frac{d_{i}}{d}+I_{v} \\
& I_{v, i}=I_{i} \times \int_{h_{i}}^{h_{i+1}} \operatorname{Chap}\left(h_{0, \mathrm{i}}, H_{i}, h\right) d h=I_{i} C_{i} \\
& I_{\text {slant }}=\sum_{i=1}^{n} I_{v, i} \times Q_{i} \\
& Q_{i}=\frac{1}{\sqrt{1-\left(\frac{\left(h_{R X}+R_{e}\right) \cos (\mathrm{El})}{h_{i}+R_{e}}\right)^{2}}} \\
& x=\frac{I_{\text {slant }} d-\left(\left(d-d_{1}\right) C_{1} Q_{1}+\left(d-d_{2}\right) C_{2} Q_{2}+\mathrm{L}\right) I_{v}}{d C_{3} Q_{3}+d_{1} C_{1} Q_{1}+d_{2} C_{2} Q_{2}+d_{4} C_{4} Q_{4}+\mathrm{L}}
\end{aligned}
$$

Our algorithm divided ionosphere into multi-shell. Figure 1 is a conceptual figure of the new method. First, we get vertical ionospheric delay at reference station from the slant delay of a satellite which has the highest elevation angle at the moment. When converting the slant delay to vertical delay, we use the obliquity factor of the thin shell model. It is acceptable idea because the error of the obliquity factor is negligible when the satellite has high elevation angle. $I_{i}$ in the figure 1 means the vertical delay of ionosphere along the whole height at the point where line of sight (LOS) vector and the i'th shell meet. What we want to solve is vertical ionospheric delay at IPP, so we set it as an unknown character $x$. By assuming that the variation of ionosphere is linear, we can mapping the overall delay of the ionosphere as equation (4). Also we assumed that the distribution of vertical electron density follows the Chapman profile we can express the segment of whole ionospheric delay of i'th point as equation (5). If we multiply i'th obliquity factor of equation (7) to each segment and add them all, we can get slant ionospheric delay as a result like equation (6). Finally, by substituting equation (4), (5) into (6), we can solve the linear equation and get vertical ionospheric delay at IPP like equation (8). Obliquity factor in equation (7) can be obtained using geometric equations like the law of sines.

\section{Verification of the new method}

We verified the algorithm in the Korean region. We used reference stations of Korean Augmentation Satellite System (KASS) to get IPP data [4]. For ionosphere environment, we used IRI model. To verify our new method in various environment, we tested for 3 IPP data (2016.08.09 02h, 17h, 22h) in two different IRI model (2014.09.01 14h (UTC), 2011.10.23 14h (UTC)). We treated IRI model data as a true ionosphere environment. In this paper, it was assumed that electron of ionosphere exists from $100 \mathrm{~km}$ to $2000 \mathrm{~km}$ height. When obtaining the IPP data, we used broadcast (BRDC) as an ephemeris data to determine the orbit of global positioning system (GPS) satellites. Ionosphere was divided into every $5 \mathrm{~km}$ height and this interval was used in the new method which is suggested in this paper.
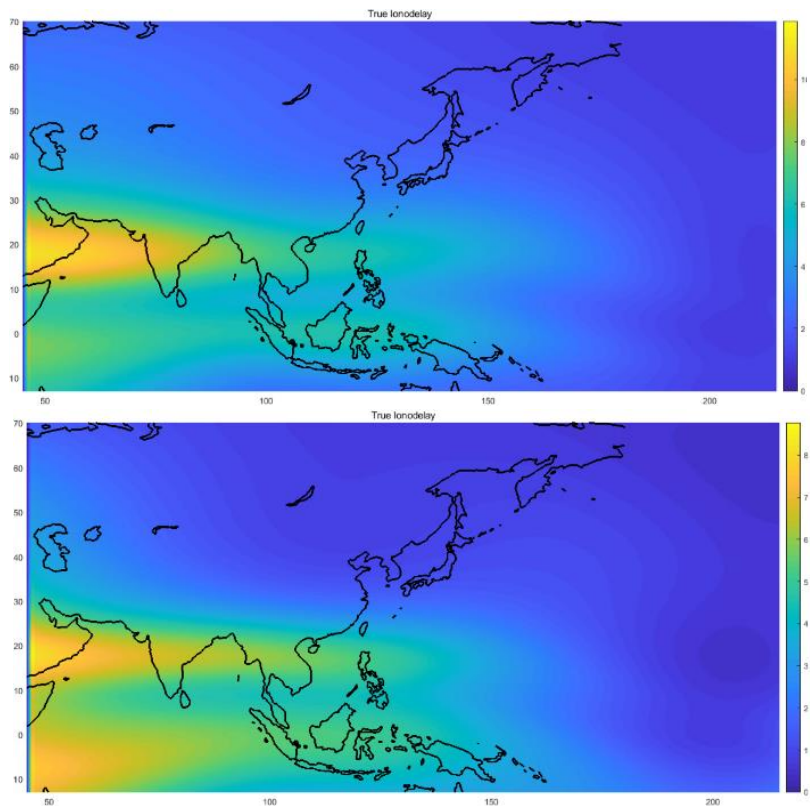

Figure 2 Ionospheric delay map

(up : 2014.09.01 14h(UTC), down : 2011.10.23 14h(UTC))

Figure 2 shows the vertical ionospheric delay of each IRI data. Because the time is $14 \mathrm{~h}$ UTC, $23 \mathrm{~h}$ for South Korea, activity around South Korea region is weak comparatively. Figure 3 and figure 4 show the result of estimation error of thin shell model and the new method. Circles in figures indicate IPPs and colors in the circle is degree of the error of each method.

As we can see in the figure 3, estimation error of thin shell model increases as IPPs are far from the Korean region where reference stations are. It means that estimation error at IPPs between the reference station and a satellite with a low elevation angle is high if the thin shell model is used. However, as we can see in the right-hand side of the figure 3, estimation error of IPPs with low elevation angle were mitigated if the new method is used. Besides overall error of estimation were mitigated. 


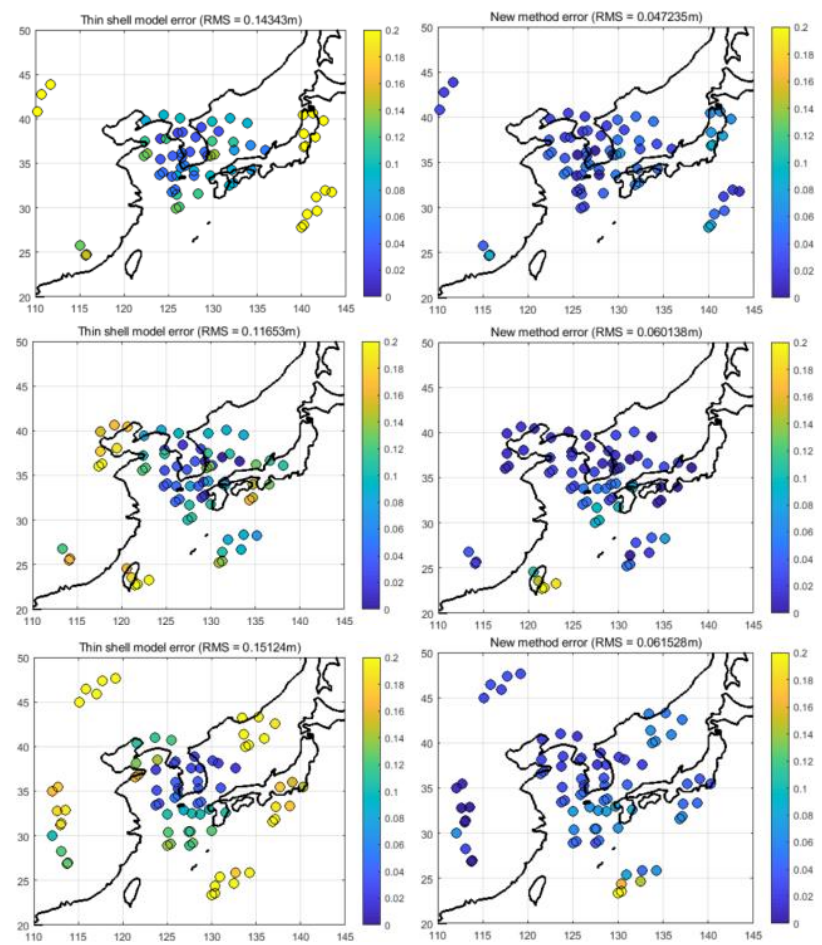

Figure 3 Estimation error at IPP (date : 2014.09.01 14h) (left : thin shell model, right : new method, up : 02h IPP data, middle : 17h IPP data, down : 22h IPP data)

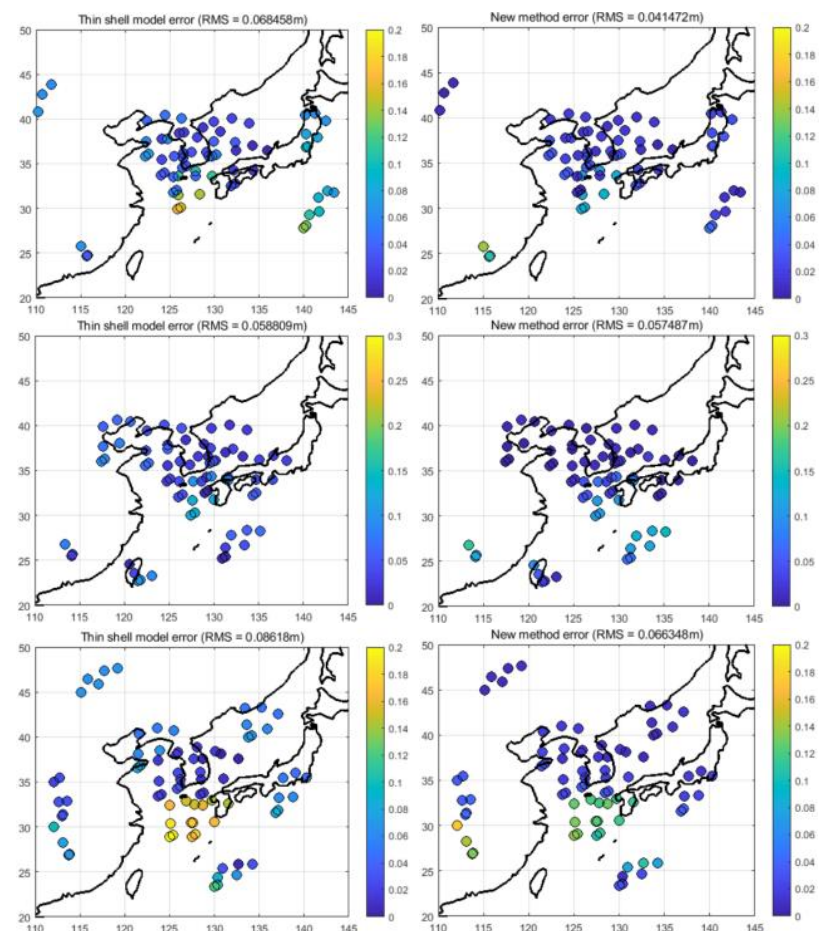

Figure 4 Estimation error at IPP (date : 2011.10.23 14h)

(left : thin shell model, right : new method,

up : 02h IPP data, middle : 17h IPP data, down : 22h IPP data)

Figure 4 shows that the estimation error is also decreased in another ionospheric environment when using the new method instead of the thin shell model. Comparing the IPPs which located at center of the map, we can see that estimation error is mitigated. It is because distance between the reference station and the satellite of IPPs at center is shorter than other IPP located at circumference. Variation of vertical ionospheric delay is can be treated linear in short distance. So estimation error of IPPs which have short distance can be more decreased when using the new method which assumed that variation of ionosphere is linear.

Table 1. Estimation error of each method (RMS)

\begin{tabular}{|c|c|c|c|}
\hline IRI data & IPP data & $\begin{array}{l}\text { Thin shell } \\
\text { model error } \\
\text { (m) }\end{array}$ & $\begin{array}{l}\text { New method } \\
\text { error } \\
(\mathbf{m})\end{array}$ \\
\hline \multirow{3}{*}{$\begin{array}{c}2014.09 .01 \\
14 \mathrm{~h}\end{array}$} & 02h & 0.1434 & 0.0472 \\
\hline & $17 \mathrm{~h}$ & 0.1165 & 0.0601 \\
\hline & $22 \mathrm{~h}$ & 0.1512 & 0.0615 \\
\hline \multirow{3}{*}{$\begin{array}{c}2011.10 .23 \\
14 \mathrm{~h}\end{array}$} & 02h & 0.0685 & 0.0415 \\
\hline & $17 \mathrm{~h}$ & 0.0588 & 0.0575 \\
\hline & $22 \mathrm{~h}$ & 0.0862 & 0.0663 \\
\hline
\end{tabular}

Table 2. Percentage of mitigated error at IPP

\begin{tabular}{c|c|c}
\hline IRI data & IPP data & Mitigated error (\%) \\
\hline \multirow{3}{*}{$\mathbf{2 0 1 4 . 0 9 . 0 1 ~ 1 4 h}$} & $\mathbf{0 2 h}$ & 67.09 \\
\cline { 2 - 3 } & $\mathbf{1 7 h}$ & 48.41 \\
\cline { 2 - 3 } & $\mathbf{2 2 h}$ & 59.33 \\
\hline \multirow{3}{*}{$\mathbf{2 0 1 1 . 1 0 . 2 3 ~ 1 4 h}$} & $\mathbf{0 2 h}$ & 39.41 \\
\cline { 2 - 3 } & $\mathbf{1 7 h}$ & 2.21 \\
\cline { 2 - 3 } & $\mathbf{2 2 h}$ & 23.09 \\
\hline
\end{tabular}

Overall result of simulation is on table 1 . In table 2 , there are percentage points mitigated error at IPP. The percentage point indicates decreased error by using the new method over the error of thin shell model. As we can see in the table 1 , estimation error was mitigated at all case. Besides the mitigated error was range from $2.2 \%$ to $67.1 \%$. The average of mitigated error was about $40 \%$.

The future work is to verify the new method in various ionosphere environment especially when the activity of ionosphere is severe. It is because when the ionosphere is severe, the assumption that ionosphere linear may occur the error more than in the weak ionosphere environment. However, with previous result, we verified that our new method works well in weak ionosphere environment.

\section{Conclusion}

In this paper we suggested the new method for converting slant ionospheric delay to vertical ionospheric delay at IPP. We used the Chapman profile which is model for vertical electron density distribution of ionosphere. Furthermore, with assumption that variation of ionosphere is linear and parameters of Chapman profile is given, the slant delay can be expressed in linear equation of vertical delay at IPP. Verification was performed with simulations which are consist of two IRI model sets and three IPP data sets. As a result, the estimation error at IPP was mitigated about $40 \%$ on average when using the new method instead of the thin shell model. 


\section{Acknowledgement}

This research was supported by Basic Science Research Program through the National Research Foundation of Korea(NRF) funded by the Ministry of Science, ICT \& Future Planning (No. NRF-2017M1A3A3A02016230), contracted through by the Institute of Advanced Aerospace Technology at Seoul National University. The Institute of Engineering Research at Seoul National University provided research facilities for this work.

\section{Reference}

[1] Hoque, M. M., and N. Jakowski. "A new global model for the ionospheric F2 peak height for radio wave propagation." Annales Geophysicae. Vol. 30. No. 5. Copernicus GmbH, 2012

[2] Feltens, J. "Chapman profile approach for 3-D global TEC representation." IGS Presentation, in (1998).

[3] Hoque, M. M., N. Jakowski, and J. Berdermann. "A new approach for mitigating ionospheric mapping function errors." Proceedings ION GNSS. 2014.

[4] Authié, T., Dall'Orso, M., Trilles, S., Choi, H., Kim, H., Lee, J., Lee, E., Nam, G., "Performances Monitoring and Analysis for KASS," Proceedings of the 30th International Technical Meeting of The Satellite Division of the Institute of Navigation (ION GNSS+ 2017), Portland, Oregon, pp. 958978, September 2017.

[5] Hoque, M. M., and N. Jakowski. "Mitigation of ionospheric mapping function error." Proceedings of the ION GNSS+, Nashville/Tennessee/USA (2013): 16-20. 